\title{
Global and Local Detection of Liver Steatosis from Ultrasound
}

\author{
Ricardo Ribeiro $^{1,2}$, Rui Tato Marinho ${ }^{3}$ and J. Miguel Sanches ${ }^{1,4}$ Senior Member, IEEE,
}

\begin{abstract}
Liver steatosis is a common disease usually associated with social and genetic factors. Early detection and quantification is important since it can evolve to cirrhosis.

Steatosis is usually a diffuse liver disease, since it is globally affected. However, steatosis can also be focal affecting only some foci difficult to discriminate. In both cases, steatosis is detected by laboratorial analysis and visual inspection of ultrasound images of the hepatic parenchyma. Liver biopsy is the most accurate diagnostic method but its invasive nature suggest the use of other non-invasive methods, while visual inspection of the ultrasound images is subjective and prone to error.

In this paper a new Computer Aided Diagnosis (CAD) system for steatosis classification and analysis is presented, where the Bayes Factor, obatined from objective intensity and textural features extracted from US images of the liver, is computed in a local or global basis. The main goal is to provide the physician with an application to make it faster and accurate the diagnosis and quantification of steatosis, namely in a screening approach.

The results showed an overall accuracy of $93.54 \%$ with a sensibility of $95.83 \%$ and $85.71 \%$ for normal and steatosis class, respectively. The proposed CAD system seemed suitable as a graphical display for steatosis classification and comparison with some of the most recent works in the literature is also presented.
\end{abstract}

\section{INTRODUCTION}

Fatty liver infiltration, called steatosis, occurs when the fat content of the hepatocytes increases [1]. Worldwide, the prevalence of hepatic steatosis has increased, in great part associated with obesity, insulin resistance and alcohol [2].

Early detection is of major clinical importance, since steatosis is the first biomarker for the potential development of cirrhosis and even hepatocellular carcinoma [2].

Liver biopsy remains the reference exam for the evaluation of hepatic steatosis [2]. The need for biopsy reduction, due to its invasive nature and the potential of sampling errors, has led to the development of non-invasive methods for steatosis diagnosis.

Among the new techniques for liver steatosis assessment, imaging methods are widely reported in the literature. The systematic review study of [2] shows that magnetic resonance

\footnotetext{
This work was supported by the FCT project [PEstOE/EEI/LA0009/2011]

${ }^{1}$ Institute for Systems and Robotics, IST, Lisbon, Portugal.

${ }^{2}$ Escola Superior de Tecnologia da Saúde de Lisboa, Lisbon, Portugal.

${ }^{3}$ Liver Unit, Department of Gastroenterology and Hepatology, Hospital de Santa Maria, Medical School of Lisbon, Portugal

${ }^{4}$ Department of Bioengineering, Instituto Superior Técnico / Technical University of Lisboa, 1040-001 Lisbon, Portugal

Emails: Ricardo Ribeiro: ricardo.ribeiro at estesl.ipl.pt, Rui Tato Marinho: rui.marinho at mail.telepac.pt, J. Miguel Sanches: jmrs at ist.utl.pt
}

978-1-4577-1787-1/12/\$26.00 @2012 IEEE imaging (MRI) performs better than ultrasound (US) and computed tomography (CT). Nevertheless, drawbacks such as exam cost and ionizing nature are shown in MRI and CT, respectively. On the contrary, US shows a non-invasive, low price, accessible, and non-ionizing nature.

The accumulation of fat in the liver leads to an increase of brightness and changes on the textural characteristics of the US images of the liver parenchyma. However, the source of these changes may be in reflective echoes arising from other hepatic diseases.

A large spectrum of studies has debated the optimal and sub-optimal features extracted from US images for steatosis classification purposes. In this scope, a set of features extracted from the US liver parenchyma images are used to detect the steatosis condition. The most common features described in the literature are based on the first order statistics [3], [4], co-occurrence matrix [5], wavelet transform [6], [5], attenuation along the depth [7] and backscattering [7] parameters and coefficients. Also, in this study, it is compared the results of four different classifiers: Bayes, $\mathrm{k}$ nearest neighbor $(\mathrm{kNN})$, Support Vector Machines (SVM): polynomial and radial-basis kernels.

The development of the CAD system is here presented based on the feature extraction/selection and classification from US images. For this reason an improvement in the usual US examination is attempted in this study. Moreover, this CAD system is organized has a graphical interface, in which the classification result is embedded in the original US image, allowing a local fat analysis of the liver parenchyma.

This paper is organized as follows. Section II formulates the problem, describes the image acquisition procedures, the extraction and selection of features and the classifiers used. Section III describes the experimental tests and presents the classification results. It is also discussed the results from the proposed CAD interface system. Section IV concludes the paper.

\section{Materials And Methods}

In a US pattern recognition problem, it is necessary to assure the objectivity and reproducibility of image acquisition before feature extraction and classification.

In this sense, an ultrasound machine preset was established by using a fundamental frequency of $3.5 \mathrm{MHz}$, image depth of $18 \mathrm{~cm}$, two focal zones were set at the central portion of the image $(9 \mathrm{~cm})$ and the dynamic range set in $75 \mathrm{~dB}$. Gain was variable, according to the patient biotype, and 
the depth gain compensation was set to its central position and kept constant throughout the examinations. US images were acquired only from the right liver lobe and the same anatomical landmarks were used, in order to standardize the acquisition protocol.

Images were stored in the Digital Imaging and Communications in Medicine (DICOM) format. From each US image, a region-of-interest (ROI) of approximately $100 \times 100$ pixels along the medial axis was extracted, using as criteria: i) ROI sample should be visually representative of the liver parenchyma; ii) major blood vessels and liver ligaments should be avoid; and iii) the sample should be as superficial as possible, to avoid US beam distortions.

Two important US features are used in clinical practice: liver parenchyma echogenicity and its texture. According to the studies [7], [8], [6], [5], [9], [10], the following features were extracted. From the echogenicity point of view, the acoustic attenuation coefficient is studied by means of the slope of the linear regression of the mean image intensity along the depth direction (lines).

In this work the attenuation coefficient, $\mathrm{m}$, is estimated by using the method proposed in [7]. This is addressed as the following linear regression problem

$$
\hat{m}=\arg \min _{m} \mathcal{J}(\boldsymbol{X}),
$$

where $\boldsymbol{X}$ is the $N \times M$ US liver image. The objective function is

$$
\mathcal{J}=\sum_{l=0}^{N}(\alpha l+b-f(l))^{2}
$$

where $f(l)=\frac{1}{M} \sum_{c=1}^{M} x_{l, c}$ is the average value intensity of each image line, in the assumption, that depth increases along each column.

From the textural point of view, two main features categories were extracted: I) using the wavelet transform, the vertical, horizontal and diagonal detail components are extracted, of the first and second Haar wavelet decomposition [11], and from it the energy and mean are computed; II) the autoregressive (AR) model coefficients, $\theta=\left\{a_{1,1}, a_{1,0}, a_{0,1}\right\}$, corresponding to the coefficients of the first order $2 D \mathrm{AR}$ model describing the image texture .

In the canonical definition of a 1D $p$-order AR model each sample is modeled as a linear combination of the previous $p$ samples with unknown coefficients, $a_{k}$ [12]

$$
x(l)=\sum_{i=1}^{p} a_{i} x(l-i)+r(l)
$$

where the residue signal, $r(l)$, is assumed to be white and zero mean normal distributed noise. For image applications the following 2D formulation of the $(p, q)$-order AR model is used [13]

$$
x(l, c)=\sum_{i=0}^{p} \sum_{j=0}^{q} a_{i j} x(c-i, l-j)+r(l, c)
$$

where $x(l, c)$ is the ${ }^{l, c}$ th pixel of the image and $a_{0,0}=0$. The first order model was adopted, as confirmed by [14].
A total of 36 features are extracted from the ROI US image and wavelet decomposition components, for each patient.

For comparison purposes, we also extracted the features suggested by [8], namely the median, standard deviation and inter-quartile range extracted from each coefficient from the 2 levels of the wavelet packet transform using the Daubechies 3 wavelet and the original ROI.

Four types of classifiers, kNN, Bayes and SVM (polynomial and radial-basis kernel) [15], are tested with three different sub-set of features: i) wavelet-based features proposed by [8]; ii) using only the acoustic attenuation coefficient, as used in [9], [10]; and iii) the set of features proposed in this study, selected by the sequential forward floating selection method [16].

Different parameterizations were tested: for the $\mathrm{kNN}$, we tested from $k=1, \ldots, 9$ neighborhood configurations; for the SVM polynomial kernel the cost (c) ranging from 1,10,50, 100, 150 and degree (d), $d=1, \ldots, 10$; and for SVM with radial-basis kernel the $\mathrm{c}$ parameter with the same range and the radius ( $\mathrm{r}$ ) from $r=0.2, \ldots, 10$ with steps of 0.2 . Only the best results are shown in this paper.

The classifiers result, according to the three sub-set of features, are analyzed by means of the overall accuracy (OA), sensibility ( $\mathrm{Se}$ ) and specificity $(\mathrm{Sp})$, in a leave-one-out crossvalidation basis. OA is weighted by class frequencies.

After the selection of the best feature set / classifier combination, the Bayes factor is used to quantify the confidence level of the classification, healthy or steatotic, in a global and local basis. The Bayes factor is defined as follows,

$$
\Lambda=\frac{g_{F}(\mathbf{x})}{g_{N}(\mathbf{x})}
$$

A confidence map image, $\mathbf{S}=\{\Lambda(i, j)\}$, is computed where each element is obtained from a small window centered at each $(i, j)^{\text {th }}$ pixel. This map aims at to detect foci fat accumulation locations at the parenchyma and it is displayed by using a color code image overlapped with the original US image, diagnose map, $\mathbf{D}$, according with the following law,

$$
d(i, j)= \begin{cases}\alpha \Lambda(i, j+(1-\alpha) x(i, j) & \text { if } s(i, j)>0 \\ x(i, j) & \text { otherwise }\end{cases}
$$

where $x(i, j)$ is the original US image and $\Lambda(i, j)$ is the Bayes factor at the $(i, j)^{t h}$ pixel. $\alpha$ is a weight parameter to adjust the transparency.

\section{EXPERIMENTAL RESULTS}

Seventy-five (75) US liver images, obtained from 75 patients, were used in the classification process. Thirty-five (35) patients had steatotic liver and 40 had normal liver. The US images were acquired by expert operators in a Hospital facility. The selected patients had known diagnosis based on liver biopsy results. The study protocol was approved by the Ethics Committee of the Hospital, it was explained to the patients and an informed consent was obtained in each case.

As described three feature sets have been tested with four different classifiers: $A$ is the feature set proposed by 
TABLE I

BEST ACHIEVED DETECTION RATE (DR) AND OA FOR EACH TESTED CLASSIFIER WITH THE THREE DIFFERENT FEATURE SET PROPOSED.

\begin{tabular}{|c|c|c|c|c|}
\hline \multirow{2}{*}{ Feature Set } & \multirow{2}{*}{ Classifier } & \multicolumn{3}{|c|}{ Results } \\
\hline & & Normal (\%) & Steatosis (\%) & OA (\%) \\
\hline \multirow{4}{*}{$\mathbf{A}$} & Bayes & $\overline{95.83}$ & 64.28 & 88.7 \\
\hline & kNN & 100 & 78.57 & 95.16 \\
\hline & $\mathbf{S V M}_{\text {poly }}$ & 93.75 & 71.42 & 88.7 \\
\hline & $\mathbf{S V M}_{\text {radial }}$ & 87.5 & 78.57 & 85.7 \\
\hline \multirow{4}{*}{ B } & Bayes & 42.85 & 64.58 & 59.67 \\
\hline & kNN & 71.42 & 85.41 & 82.25 \\
\hline & $\mathbf{S V M}_{\text {poly }}$ & 93.75 & 50 & 83.87 \\
\hline & $\mathbf{S V M}_{\text {radial }}$ & 87.5 & 78.57 & 85.7 \\
\hline \multirow{4}{*}{ C } & Bayes & 95.83 & 85.71 & 93.54 \\
\hline & kNN & 91.66 & 57.14 & 83.87 \\
\hline & $\mathbf{S V M}_{\text {poly }}$ & 85.41 & 71.42 & 82.25 \\
\hline & $\mathbf{S V M}_{\text {radial }}$ & 87.5 & 57.14 & 80.64 \\
\hline
\end{tabular}

[8], Feature set $\mathrm{B}$ correspond to the acoustic attenuation coefficient, proposed originally by [7] and feature set C that is the result of the feature selection procedure in which we have incorporate the AR coefficients, extracted from the wavelet detail decompositions.

From the original 36 extracted features only 6 were selected, which formed feature set $\mathrm{C}$. All features are computed from the wavelet coefficients, from both levels, and are mainly related with the AR coefficients, energy and pixel mean, as shown in Figure 1. Individually, each feature shows some overlapping between the considered classes.

Organized by the 3 feature sets, Table I resumes the classification results. From feature set A, the best result was achieved by using a $\mathrm{kNN}$ classifier $(\mathrm{k}=3)$, which revealed an OA of $95.16 \%$, with an optimal result for the normal class, $100 \%$, but with a low detection rate for the steatosis class, $78.57 \%$. In B the best results only attained $85.7 \%$ in the OA with the SVM classifier, radial-basis kernel $(r=1.2$ and $c=100$ ). From this experience, the best achieved result was observed for feature set $\mathrm{C}$, with the Bayes classifier, performing an OA of $93.54 \%$ with a detection rate of $95.83 \%$ and $85.71 \%$ for normal and steatosis class, respectively.

In the study performed by [8], using a SVM classifier in a leave-one-out cross validation approach, an OA of $95.6 \%$ is achieved with sensitivity values for normal and steatosis classes being $97.4 \%$ and $93.3 \%$, respectively. Similar results were also obtained by different studies [9], [7], [17]. The present study is in accordance with this literature and similar results were also obtained here. Based on these, enough sensitivity is achieved to develop the graphical interface, towards the CAD system.

We computed the Bayes factor, using the Bayes classifier with feature set $\mathrm{C}$, to form the diagnose map, $\mathbf{D}$, displayed in Figure 2. The Bayes factor and its graphical representation, could be a helpful indicator for the quantification and localization of the main foci of fat accumulation within the liver parenchyma, in the sense that it allows a graphical correlation between the classification results and their localization in the original US image.

Figure 2 displays the algorithm results of US images

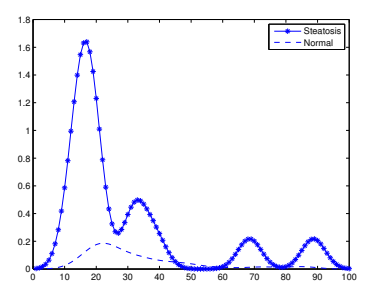

(a)

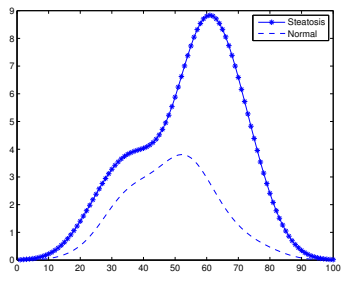

(c)

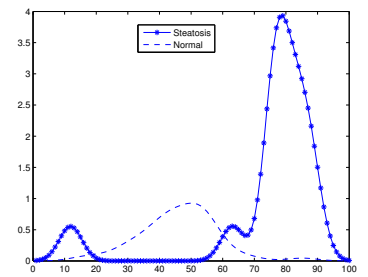

(e)

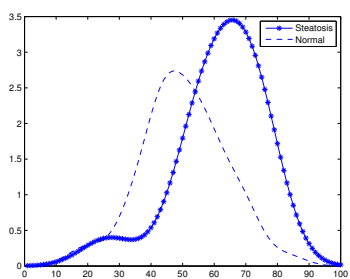

(b)

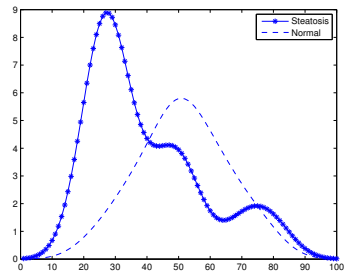

(d)

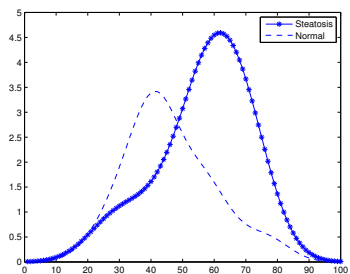

$(f)$
Fig. 1. Estimated density distribution plots obtained for each of the selected feature: (a) Energy of the wavelet horizontal detail coefficient, level 1; (b) $a_{1,0}$ and (c) $a_{0,1}$ calculated from the horizontal detail coefficient, level 2 ; (d) $a_{0,1}$ from the diagonal detail coefficient, level 1 ; (e) mean and (f) $a_{1,1}$ of the vertical detail coefficient, level 2.

from one healthy and steatotic liver sample, respectively. The resultant map images, confidence and diagnose, show that the yellow to red color regions indicate high probability of fat presence, the green region indicates that there aren't enough evidence to support a decision and the light to dark blue regions indicate normal liver tissue. These results show that the physician can select an US ROI, within the area corresponding to the liver parenchyma, and obtain a confidence map image that illustrate the local classification results, based on the Bayes factor. The diagnose map images allow the physician to have an overall relationship between the local classification results and the original US ROI, as well as gives a notion of the spatial relationship of the selected ROI with the rest of the anatomical structures.

\section{CONCLUSIONS}

In this paper a new CAD system for steatosis analysis is presented, developed from the US-based features, extracted from the wavelet transform detail coefficients, by using a Bayes classifier.

Among the four classifiers tested with three different sets of features, the best result, in a leave-one-out basis, led to an OA of $93.54 \%$ with a sensibility of $95.83 \%$ and $85.71 \%$ for normal and steatosis class, respectively. 

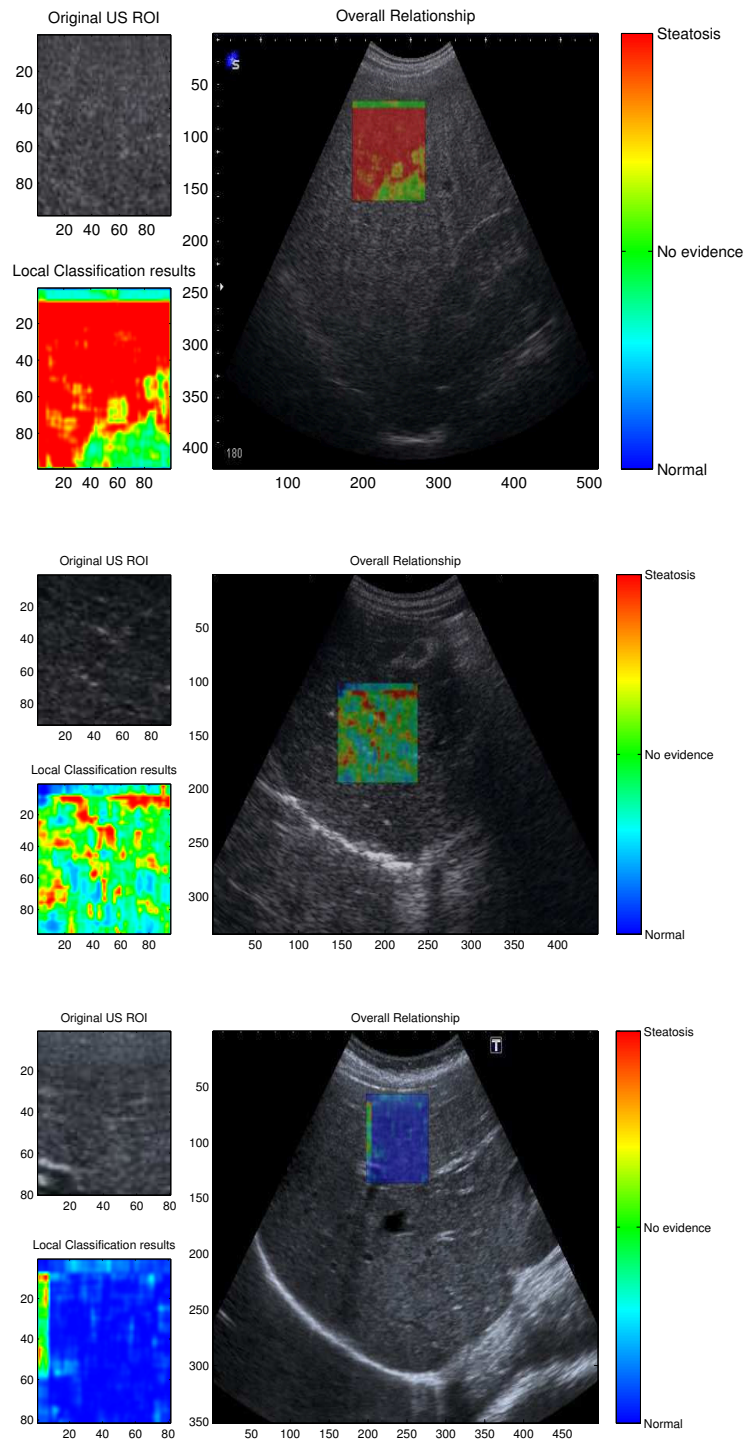

Fig. 2. Classification results displayed in the developed CAD system interface. From top to bottom, it is observed a steatotic sample with the correct classification result (steatotic), a steatotic sample that, due to lack of evidence to support the decision, it is not well classified and in the bottom a normal US liver sample that is also correctly classified.

An important result from this preliminary study, was that the AR coefficients, extracted from the multi-scale Haar wavelet decomposition, are particular relevant for the classification of liver steatosis by US images.

Based on the results, global and local assessment of liver tissue described by means of the Bayes factor can provide useful information to the physician about the confidence of the classification as well as the classification itself.

Further studies are needed, particularly, with higher number of patients, as well as with more correlation between clinical features and US-based features. Another issue is that in clinical practice it is important to discriminate between different steatosis stages. For further studies, this topic will be attempt by changing the classification and display schema to handle multiple classes.

\section{REFERENCES}

[1] S. Sherlock and J.Dooley, Diseases of the liver and Biliary System, 11st ed. Blackwell Science Ltd, 2002.

[2] A. Bohte, J. van Werven, S. Bipat, and J. Stoker, "The diagnostic accuracy of us, ct, mri and h-mrs for the evaluation of hepatic steatosis compared with liver biopsy: a meta-analysis," European Radiology, vol. 21, pp. 87-97, 2011.

[3] K. Maeda, M. Utsu, and P. E. Kihaile, "Quantification of sonographic echogenicity with grey-level histogram width: A clinical tissue characterization," Ultrasound in Medicine \& Biology, vol. 24, no. 2, pp. $225-234,1998$.

[4] C. H. Lee, J. W. Choi, K. A. Kim, T. S. Seo, J. M. Lee, and C. M. Park, "Usefulness of standard deviation on the histogram of ultrasound as a quantitative value for hepatic parenchymal echo texture; preliminary study," Ultrasound in Medicine \& Biology, vol. 32, no. 12, pp. 1817 - 1826, 2006

[5] W. Yeh, Y. Jeng, C. Li, P. Lee, and P. Li, "Liver fibrosis grade classification with b-mode ultrasound," Ultrasound in Medicine \& Biology, vol. 29, pp. 1229-1235, 2003.

[6] A. Mojsilovic, S. Markovic, and M. Popovic, "Characterization of visually similar diffuse diseases from b-scan liver images with the nonseparable wavelet transform," Image Processing, International Conference on, vol. 3, p. 547, 1997.

[7] D. Gaitini, Y. Baruch, E. Ghersin, E. Veitsman, H. Kerner, B. Shalem, G. Yaniv, C. Sarfaty, and H. Azhari, "Feasibility study of ultrasonic fatty liver biopsy: Texture vs. attenuation and backscatter," Ultrasound in Medicine \& Biology, vol. 30, no. 10, pp. 1321 - 1327, 2004.

[8] F. Minhas, D. Sabih, and M. Hussain, "Automated classification of liver disorders using ultrasound images," Journal of Medical Systems, pp. 1-10, 2011.

[9] M. Lupsor, R. Badea, C. Vicas, S. Nedevschi, M. Grigorescu, C. Radu, H. Stefanescu, and D. Crisan, "Non-invasive steatosis assessment in nash through the computerized processing of ultrasound images: Attenuation versus textural parameters," in Automation Quality and Testing Robotics (AQTR), 2010 IEEE International Conference on, vol. 2, may 2010 , pp. $1-6$.

[10] J. Thijssen, A. Starke, G. Weijers, A. Haudum, K. Herzog, P. Wohlsein J. Rehage, and C. De Korte, "Computer-aided b-mode ultrasound diagnosis of hepatic steatosis: a feasibility study." IEEE Trans Ultrason Ferroelectr Freq Control., vol. 55, no. 6, pp. 1343-54, 2008.

[11] S. Bow, Pattern Recognition and Image Preprocessing, 2nd ed. Marcel Dekker,Inc., 2002.

[12] K. Wear, R. Wagner, and B. Garra, "A comparison of autoregressive spectral estimation algorithms and order determination methods in ultrasonic tissue characterization," IEEE Transactions on Ultrasonics, Ferroelectrics and Frequency Control, vol. 42, no. 4, pp. 709-716, July 1995.

[13] P. Stathaki and A. Constantinides, "Robust autoregressive modeling through higher order spectral estimation techniques with applications to mammography," Conference Record of The Twenty-Seventh Asilomar Conference on Signals, Systems and Computers, vol. 1, pp. 189193, November 1993

[14] J. Bleck, U. Ranft, M. Gebel, H. Hecker, M. Westhoff-Bleck, C. Thiesemann, S. Wagner, and M. Manns, "Random field models in the textural analysis of ultrasonic images of the liver," Medical Imaging, IEEE Transactions on, vol. 15, no. 6, pp. 796-801, Dec 1996.

[15] S. Theodoridis and K. Koutroumbas, Pattern Recognition, Fourth Edition, 4th ed. Academic Press, 2008

[16] P. Pudil, J. Novovicová, and J. Kittler, "Floating search methods in feature selection," Pattern Recognition Letters, vol. 15, no. 11, pp. 1119-1125, 1994.

[17] M. Lupsor, R. Badea, S. Nedevschi, D. Mitrea, and M. Florea, "Ultrasonography contribution to hepatic steatosis quantification. possibilities of improving this method through computerized analysis of ultrasonic image," Automation, Quality and Testing, Robotics, 2006 IEEE International Conference on, vol. 2, pp. 478-483, May 2006. 